\title{
GUI Based Execution of Discourse Upgrade System
}

\author{
K Ashok kumar, A Karthik
}

\begin{abstract}
Discourse, being a key method for correspondence, has been inserted in different applications. In numerous unavoidable circumstances, we are rendered vulnerable attempting to conclude the understandability of the discourse and this is the place Speech improving strategy i.e. evacuation of undesirable foundation commotion, comes into picture. In this paper, an endeavor has been made towards contemplating Speech Enhancement methods, for example, Spectral Subtraction, Minimum Mean Square Error (MMSE), Kalman and Wiener channel. In view of our perceptions and investigation of different execution parameters, we finish up which of the strategies is most reasonable for discourse improvement. The usage of the code for different channels is finished utilizing.
\end{abstract}

Keywords- Kalman filter, MMSE, SNR, Spectral subtraction, Speech enhancement, Wiener filter

\section{INTRODUCTION}

Discourse is an essential and regular medium, subsequently vital for us, to convey. Progressions in innovation have cleared a path for some, more discourse situated applications like cell voice calls, VoIP, remotely coordinating frameworks, discourse acknowledgment, and amplifiers, and so on. As a rule, these frameworks function admirably in almost clamor free conditions, however their execution decays quickly in uproarious conditions. When all is said in done, there exists a need to build the unwavering quality of these frameworks in loud conditions. In this way, enhancement in existing pre-preparing calculations or presenting whole new class of calculation for discourse improvement is the essential target of research network. In discourse upgrade, the objective is to enhance the nature of debased discourse. Discourse upgrade calculations are commotion concealment methods, utilizing the information from the field of hearing science, that alleviate the impact of the defiling foundation clamor, and subsequently enhance the apparent discourse quality and discourse coherence.

The issue of enhancing execution of discourse correspondence frameworks in boisterous conditions has been a testing zone for research for over three decades now. For making discourse coders strong to clamor, discourse improvement centers around enhancing the quality and comprehensibility of discourse harmed by commotion. However, on the off chance that discourse is over prepared, clarity of uproarious discourse may diminish quickly frustrating powerful enhancement in upgrading the execution of other discourse applications, for example, discourse

Revised Manuscript Received on September 22, 2019.

K Ashok kumar, Assoc. Professor, Dept. of ECE, Malla Reddy Engineering College for Women(Autonomous), Secunderabad-TS akkonduru@gmail.com

A Karthik, Asst. Professor, Dept. of ECE Narasimha Reddy Engineering College, Hyderabad, Telangana State, India

Karthik011190@gmail.com coding/pressure and discourse acknowledgment [1-4]. This venture introduces a diagram of various discourse upgrade. Utilizing tables and diagrams we think about and audit the systems.

\section{TYPES OF SPEECH ENHANCEMENT}

Discourse Enhancement techniques can be grouped from various perspectives. A standard calculation alone is deficient for every one of the kinds of clamor present in the encompassing. In this way, discourse improvement calculations are created dependent on the applications. The suspicions and different limitations are resolved totally as per the application and the earth. The execution of discourse improvement calculation is constrained by variables like number of clamor sources accessible, constraints in time varieties and accessible from the earlier information. Demonstrate based requirements like limitation of calculation to uncorrelated commotion likewise assume an essential job. The Speech Enhancement frameworks can be characterized dependent on number of channels utilized i.e. single or numerous, space of preparing i.e. time or recurrence and the kind of calculations. Discourse handling systems can be extensively partitioned into single and multichannel upgrade procedures [5-6].

\section{A. Single channel discourse improvement}

In applications like portable amplifiers and cell phones, where a substitute channel is inaccessible, single channel upgrade is utilized. Single channel improvement strategies are anything but difficult to assemble and are more affordable when contrasted with its multichannel partner. A disservice of this technique is that the flag can't be pre-handled since there is no commotion reference flag present. Thus, single channel upgrade frameworks make utilization of an alternate factual way to deal with the undesirable clamor [7]. The vast majority of the single channel improvement calculations chip away at the supposition that clamor is stationary in nature.

\section{B. Multi-channel discourse upgrade}

This technique for discourse improvement is superior to the recently referenced strategy because of essence of reference channel. This channel causes us dispose of commotion in a compelling way. Stage arrangement is performed in one of the channels to dismiss the undesired commotion parts. The confinements of single channel upgrade strategy are survived and non-stationary commotion sources can be tended to in this technique for discourse improvement. Multi-channel frameworks are increasingly intricate. This strategy is actualized in versatile clamor scratch-off gadgets where a reference of debasing commotion is available in a helper channel.

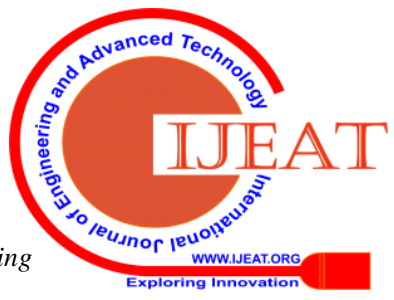




\section{GUI Based Execution of Discourse Upgrade System}

This reference input is then sifted. Commotion is thus subtracted from uproarious discourse contribution by following a versatile calculation. The principle downsides are creation cost and multifaceted nature of the calculation. This system is right now in application in cell phones for Noise Reduction utilizing Dual Mic.

\section{BASIC BLOCK DIAGRAM}

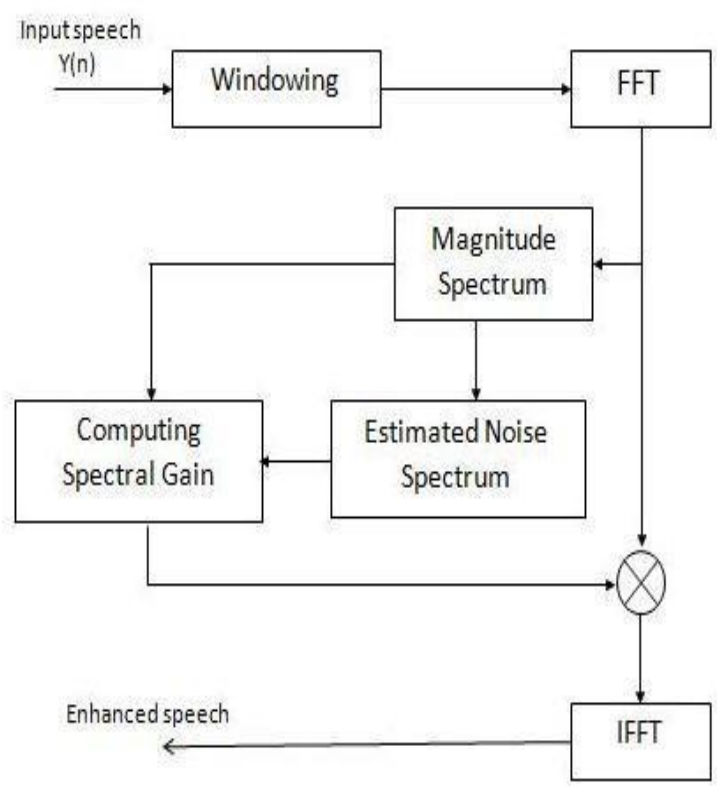

Figure 1: Basic block diagram

The loud info flag is sent through the examination window. Here, a couple of tests of the flag are chosen at once as the flag is ceaseless and enormous and can't be prepared in one go. Quick Fourier Transform is connected to change over the flag from time area to recurrence space. The greatness of clamor and boisterous discourse are thought about and commotion is subtracted from the influenced discourse. The upgraded discourse got is in recurrence space and thus requires to be changed over back to recurrence area. This is finished by taking Inverse Fourier Transform. Cover and add technique is connected to the recuperated improved flag in order to make up for the windowing strategy connected at the outset.

\section{TYPES OF FILTERS}

\section{A. Phantom Subtraction technique}

The Spectral Subtraction strategy is a standout amongst the most broadly utilized techniques for discourse improvement. This is a result of the straightforwardness of usage and lower computational load. The power range or greatness Spectrum of the discourse flag can be effortlessly reestablished utilizing approach. The evaluated clamor range is subtracted from the boisterous discourse contribution to request to acquire clean discourse. It decreases the impact of foundation commotion dependent on the STSA estimation system [8-9].

The adequacy in upgrading the discourse flag, corrupted by clamor, makes this strategy a famous one. The fundamental guideline incorporates subtraction of size range of commotion from that of uproarious discourse. The commotion range is evaluated amid the periods when just clamor is available. The clamor is thought to be uncorrelated and added substance in nature. Since stage contortion isn't seen by human ear, it is kept unaltered.
The straightforward subtraction process maybe accompanies a cost. The subtraction procedure should be done cautiously so as to maintain a strategic distance from any mutilation. On the off chance that an excess of commotion is subtracted, some discourse data may be lost. Then again in the event that too little is subtracted, the mutilation will stay in the discourse. The square chart given in [10] is as appeared in Fig. 2.

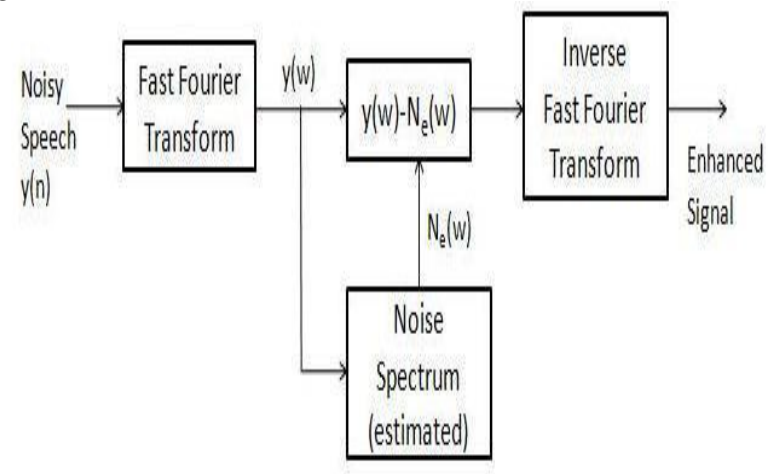

Figure 2:Spectral Subtraction

Loud Speech, $\mathrm{y}(\mathrm{n})$, is given as a contribution to this channel. Windowing is done so as to take settled number examples of the flag which is consistent in nature. In this strategy just the greatness is considered. The stage part isn't thought about as it builds the intricacy and figurings. Fourier change is connected to the flag so as to change over the flag from time space to recurrence area. This encourages us to acquire greatness and stage as independent qualities. The extent of evaluated commotion, $\mathrm{Ne}(\mathrm{w})$, is subtracted from the greatness of boisterous flag and an upgraded Speech is gotten at the yield of phantom change square. Reverse Fourier change of the improved discourse is taken in order to get the flag in its time area shape. Period of flag, in its unique shape, is added to the size at this stage. In this way we get an improved adaptation of the boisterous Speech motion at the yield end.

\section{B. Weiner Filter}

For over two decades, discourse preparing has been a developing and dynamic field. This plainly shown further development and improvement. To upgrade the channel we have to limit the mean square mistake estimation of the channel. This is finished by figuring the distinction between the ideal reaction and the real channel yield. Weiner channel is portrayed as a class of ideal direct channels [11-12]. These channels include straight estimation of an ideal flag grouping from another related arrangement. This method is broadly utilized in the field of flag handling. Weiner channel is a typical and in addition a versatile sifting method and is the answer for stationary info signals.

The channel previously began in Kalman's record (1960). In the record [13], it is portrayed as a recursive answer for the straight sifting issues. Estimation of qualities through the recursive minimum squares depended on state-space models. In view of various application necessities, a Weiner channel is intended to upgrade the flag for that extremely wanted recurrence reaction. $\mathrm{Be}$ that as it may, before the real preparing, the phantom properties of the first flag and clamor ought to be known. 


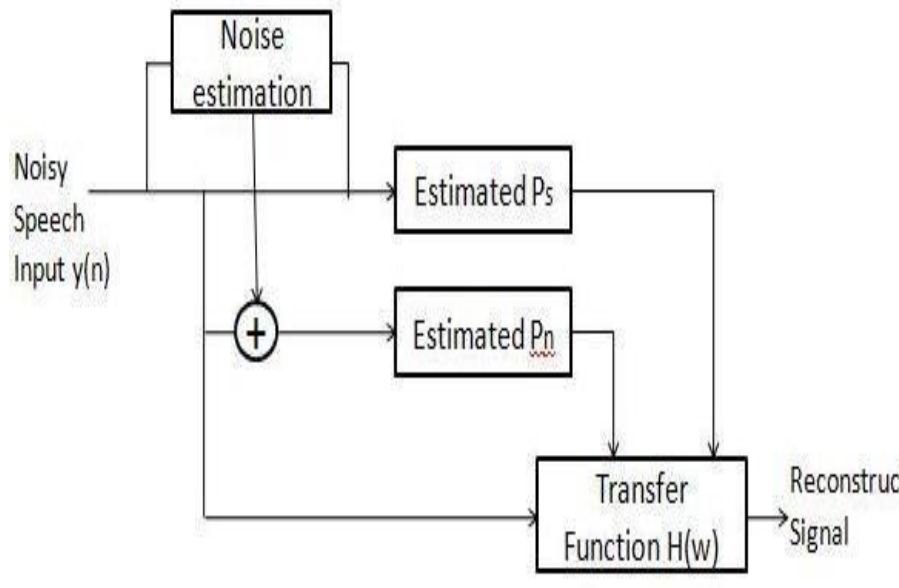

Figure 3: Weiner filter

Shown above in Fig. 3, is the square outline of Weiner channel. In this procedure, mean the loud discourse is spoken to as $\operatorname{Ps}(w)$ and mean of clamor control is spoken to as $\mathrm{Pn}(\mathrm{w})$, which is determined for recuperating the first flag.

\section{Least Mean Square Error}

Least mean square blunder method can be actualized when the info SNR is known. This calculation limits the evaluated blunder because of both clamor and mess. It is a usage of Weiner Filter. The STSA estimation issue is to evaluate the modulus of every unpredictable Fourier development coefficient of the discourse motion from the uproarious discourse in that investigation outline [14]. Fourier development arrangement are tests of its Fourier Transform and have a nearby connection [15-17]. The connection between Fourier arrangement development and discrete Fourier change empowers utilization of FFT for effective execution of the calculation.

For execution of the MMSE STSA estimator, the from the earlier likelihood conveyance of the Speech and clamor Fourier development coefficients ought to be known. Since progressively they are obscure, every likelihood dispersion can be estimated or a sensible measurable model be expected. In this paper, the Speech and the clamor are neither stationary nor Ergodic procedures. Subsequently likelihood appropriation can be gotten by looking at autonomous example capacities having a place with the group of each procedure. In any case, due to non-stationary nature of process, likelihood appropriations are time changing, their estimation and portrayal by the above way is convoluted, and the strategy is by all accounts impracticable.

The main weakness of the MMSE processor [18] is extra intricacy in deciding the straight estimator. Further, for expansive issues, the network opposite task required to execute the MMSE estimator is exceptionally risky and can back off preparing speed, as in the field of radar flagging. Another execution of the MMSE calculation can be created and the information vector can be part into littler portions to lessen handling time. The essential square chart of MMSE channel is appeared in Fig. 4

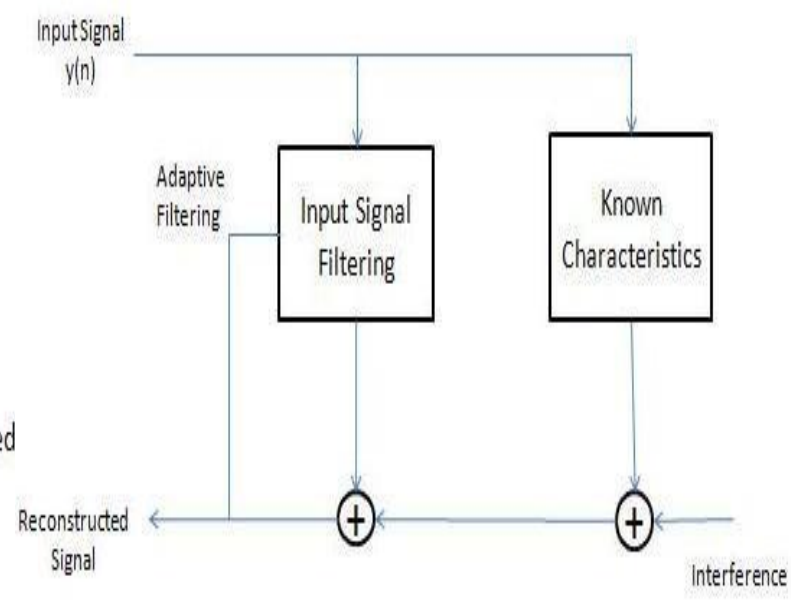

Figure 4: MMSE Filter

\section{Kalman Filter}

Kalman channel (KF) calculation depends on consistent usage of the MMSE estimator [19]. It is a lot of numerical conditions giving an effective computational intends to evaluate the condition of a procedure. Along these lines, it recursively limits the mean of the squared blunder. It utilizes state space model to convey a praiseworthy method for removing a flag from commotion. These state space models are portrayed by an Auto-Regressive (AR) process. Thus, this quick procedure can deliver pragmatic answers for issues which represented a huge obstacle in the Weiner channel.

The Kalman is an advanced channel that consequently modifies itself (when the information flag and meddling clamor change) to give you the most ideal thought of what the present condition of the framework is. The Kalman channel continues refreshing and enhancing itself by attempting to foresee the most current esteem and after that contrasting this with the watched/evaluated esteem. It doesn't straightforwardly allude to evaluated emphasis, however in truth it makes the most ideal utilization of the whole history of the information to make sense of what next estimation, regardless of the meddling commotion. The covariance framed from the forecast portrayed in [20], with another gauge each progression is rehashed with each example of information can be indicated as

It is an ideal estimator i.e. it deduces parameters of enthusiasm from equivocal, inconsistent and undefined perceptions. In the event that all commotion is White Gaussian, the Kalman channel limits the mean square mistake of the assessed parameters. Given just the mean and standard deviation of commotion, the Kalman channel is the best direct estimator. In view of a state space approach it displays flag age and the boisterous and twisted perception flag is demonstrated by a perception condition [21-22]. The utilization of Kalman channel for Speech Enhancement was first presented by Paliwal (1987). 
The upsides of Kalman Filtering Technique are great outcomes by and by because of optimality and structure. The channel is recognized by its ability to foresee the condition of a model previously, present and future, despite the fact that the correct idea of the demonstrated framework is obscure. The dynamic demonstrating of a framework is one of the key highlights which recognize the Kalman technique. In view of its clearly basic and effortlessly customized calculation, the Kalman channel will keep on assuming an essential job in GPS-based route frameworks. The disservices of Kalman Filtering Technique are that it is important to know the underlying states of the mean and change state vector to begin the recursive calculation. There is no broad assent over the method for deciding the underlying conditions.

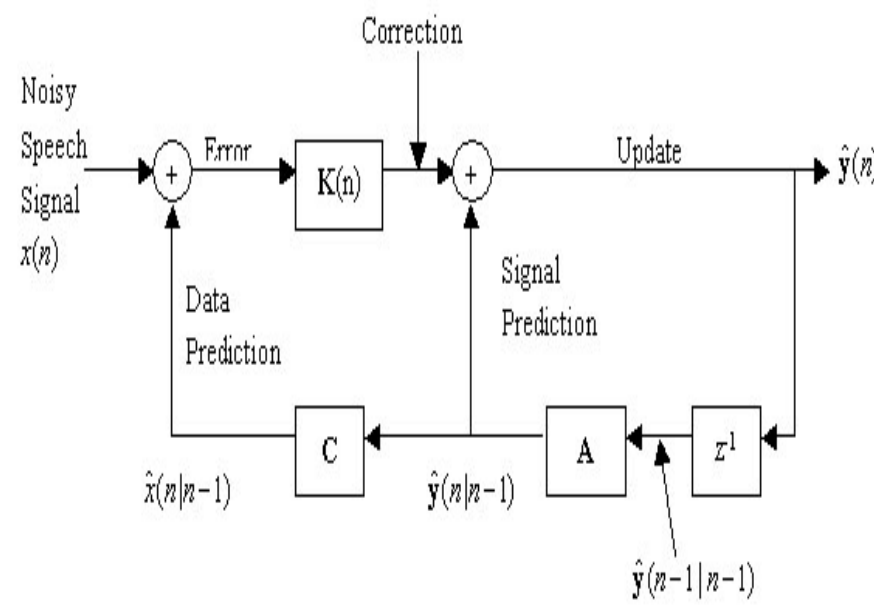

Figure 5: Kalman Filter

As appeared in Fig. 5, the info Speech flag is taken and mutilation of commotion in the flag is found.The current yield depends on the past yield and current information which is unraveled utilizing Yule's condition. Every one of the parameters are spoken to as state space framework since it makes estimations simpler. Next channel gain is determined and commotion is then expelled from the uproarious Speech contribution to get upgraded Speech signal.

\section{MEASURES OF PERFORMANCE PARAMETERS}

\section{A. Motion to-Noise Ratio (SNR)}

Motion to-clamor proportion is a measure utilized in science and building that looks at the dimension of an ideal flag to the dimension of foundation commotion as given in [3].

Motion to-commotion proportion is in some cases used to allude to the proportion of helpful data to unimportant information in a discussion or trade. Motion to-commotion proportion is characterized as the power proportion between a flag (important data) and the foundation clamor (undesirable flag). It is estimated in $\mathrm{dB}$ and is spoken to as SNR or S/N

\section{B. Mean Square Error (MSE)}

The Mean Squared Error (MSE) estimated in $\mathrm{dB}$ is one of the approaches to decide the contrast between qualities suggested by an estimator and the genuine estimations of the amount being evaluated. MSE relates to the normal estimation of the squared mistake misfortune. MSE measures the normal of the squares of the "mistakes". The contrast between the qualities inferred by estimator and the amount which is to be assessed gives us the blunder estimation of the flag. The haphazardness of flag or the powerlessness of the framework to create exact qualities at the yield offers ascend to blunder in the flag.

\section{Standardized Root Mean Square Error (NRMSE)}

The Root Mean Square Error (RMSE) otherwise called Root-Mean-Square Deviation (RMSD) is a much of the time utilized measure to discover the standardized estimation of distinction between the qualities anticipated by an estimator and the qualities that are really watched. The RMSE gives us a normal of the extent of blunders determined over some undefined time frame. RMSE is a decent proportion of exactness of a framework.

\section{Pinnacle Signal-to-Noise Ratio(PSNR)}

Pinnacle Signal-to-Noise Ratio, regularly shortened PSNR, is utilized to speak to the proportion of most extreme conceivable estimation of a flag to the intensity of the undesired clamor flag. Since numerous signs have a wide powerful range, PSNR is typically communicated as far as the logarithmic decibel scale. PSNR is commonly used to quantify the nature of recreation of lossy pressure codes. If there should be an occurrence of picture pressure, for instance, the flag is the first picture and the clamor is the mistake acquainted due with pressure. PSNR is fundamentally a surmised an incentive to the human view of the nature of remaking of discourse flag. A higher PSNR demonstrates that the remade flag has a superior quality yet the turn around might be valid if there should arise an occurrence of a few applications.

\section{E. Bending (AAD)}

Bending is fundamentally the modification or twisting of the first shape or different attributes of an item, picture, sound or waveform. Twisting is typically undesirable, and regularly endeavors are made to lessen it however much as could reasonably be expected. The expansion of commotion or other outside signs (murmur, impedance) may prompt twisting of flag. In this undertaking we utilize the parameter AAD to gauge the bending in the given Speech flag.

\section{EXPERIMENTAL RESULTS}

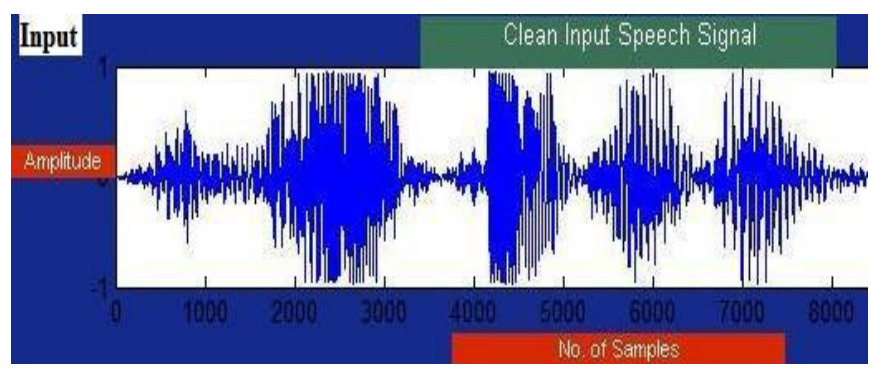

Figure 6: Clean Speech

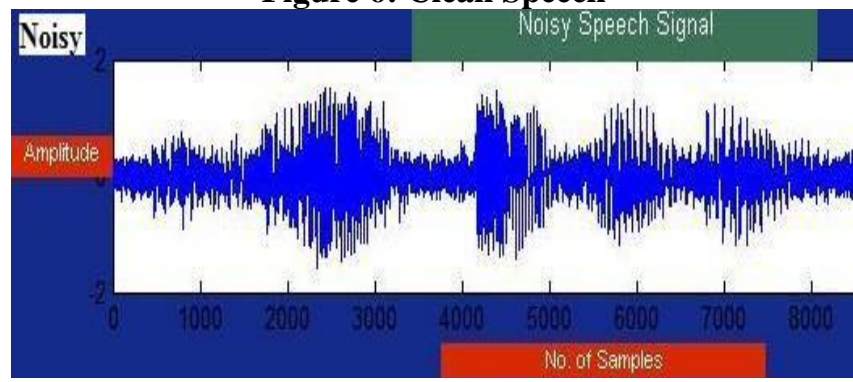

Figure 7: Noisy Speech 


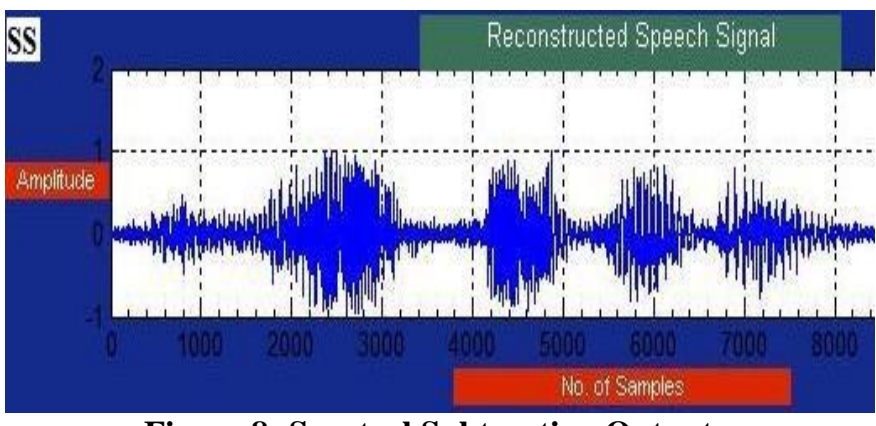

Figure 8: Spectral Subtraction Output

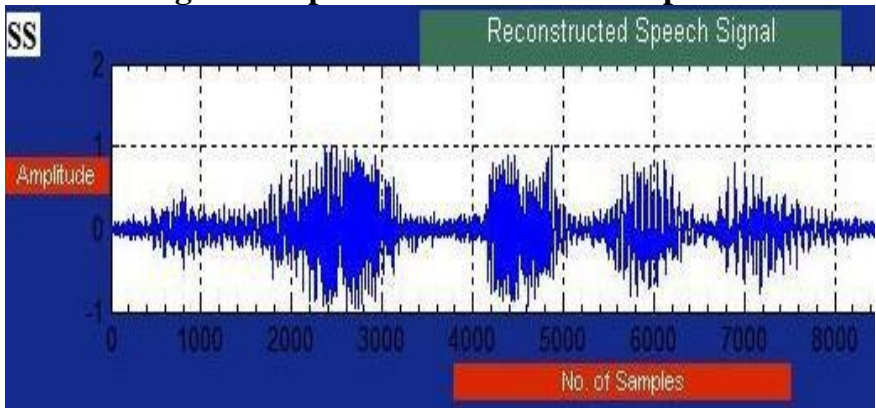

Figure 9: Weiner Filter Output

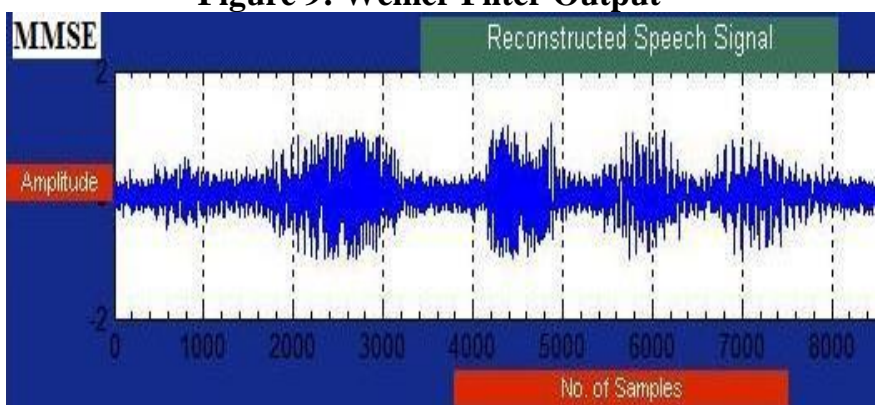

Figure 10: MMSE Filter Output

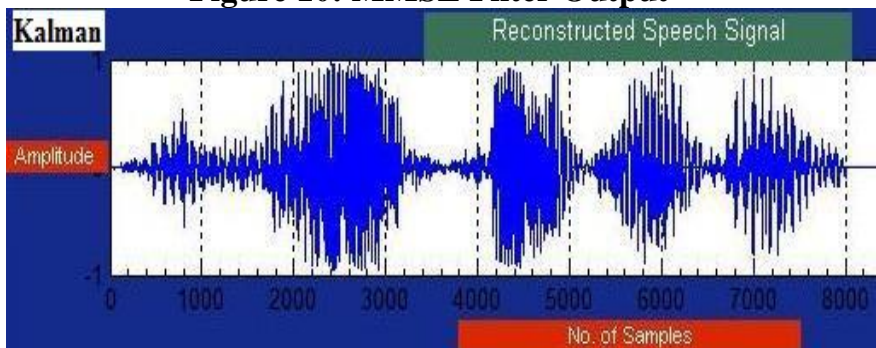

Figure 11: Kalman Filter Output

\section{CONCLUSION}

From the graphs we can clearly see and eventually conclude that MMSE filter provides the best SNR ratio which a major requirement as we cannot accept a filter degrading the original speech. It also filters out the noise and renders us with clean speech in a more flat line Peak SNR value in a varying loudness of speech. We tried out playing the reconstructed signal after processing the noisy speech using the four filters and MMSE definitely sounded better than the rest.

\section{REFERENCES}

1. Yariv Ephraim and Israel Cohen, "Recent Advancements in Speech Enhancement", Acoustics, Speech and Signal Processing, IEEE Transactions on, vol.32, no.5, pp. 1109- 1121, March 9, 2004.

2. T. Lalith Kumar and Soudara Rajan, "Speech Enhancement Using Adaptive Filters", VSRD-IJEECE, Vol. 2 (2), 2012, 92-99, 1997.

3. http://en.wikipedia.org/wiki

4. http://www.mathworks.com/support

5. Javier Ortega-Garca and Joaqun GonzlezRodrguez, "Overview of Speech Enhancement Techniques for Automatic Speaker Recognition", CICYT under Project TIC94-0030., 1999.
6. Prof. MilindNemade, Prof. Satish K. Shah, "Performance Comparison of Single Channel Speech Enhancement Techniques for Personal Communication", for International Journal of Innovative Research in Computer and Communication Engineering, Vol. 1, Issue 1, March 2013.

7. Yao Ren and Michael T. Johnson, "Improved SNR Estimator for Speech Enhancement”, ICASSP-IEEE, 2008.

8. Saeed V. Vaseghi, "Spectral Subtraction", Advanced Digital Signal Processing and Noise Reduction, Second Edition, 1996.

9. MukulBhatnagar, "A modified spectral subtraction method combined with perceptual weighting for speech enhancement", Thesis presented to The University of Texas, August 2002.

10. J.Surendiran1 ， Dr.S.V.Saravanan , F.Elizabeth Catherine," GLAUCOMA DETECTION USING FUZZY C- MEAN (FCM)", IJPT| Sep-2016 | Vol. 8 | Issue No.3 | 16149-16163.

11. M. A. Abd E-Fattah, M. I. Dessouky, S. M. Diab and F. E. Abd El-Samie, "Speech Enhancement using Adaptive Weiner Filtering Approach", Progress in Electromagnetic research M, Vol. 4, pp. 167-184, 2008.

12. Bram Cornelis, Marc Moonen and Jan Wouters, "Performance Analysis of Multichannel Wiener \& Filter-Based Noise Reduction in Hearing Aids Under Second Order Statistics Estimation Errors", IEEE Transactions on Audio, Speech and Language Processing, VOL. 19, No. 5, July 2011, 2011.

13. Kalman, R. E. 1960. "A New Approach to Linear Filtering and Prediction Problems," Transaction of the ASME-Journal of Basic Engineering, pp. 35-45, March 1960.

14. Dr. Bin Chen, "Speech Enhancement using a Laplacian based MMSE Estimator of the magnitude spectrum", 2005.

15. Ephraim, Y., Malah D., "Speech enhancement using a Minimum mean square error short-time spectral amplitude estimator," Acoustics, Speech and Signal Processing, IEEE Transactions on , vol.32, no.6, pp. 11091121, Dec 1984

16. Bin Chen, Philipos C. Loizou, "A Laplacian based MMSE estimator for speech enhancement", December 20, 2006.

17. Peter Kabal, "Minimum Mean-Square Error Filtering Autocorrelation/Covariance, General Delays, and Multirate Systems", April 2011.

18. Deepa, "Dual channel speech enhancement using Hadamard LMS algorithm with DCT pre-processing technique", International Journal of Engineering Science and Technology, Vol. 2(9), 2010.

19. Ning Ma, Martin Bouchard and Rafik A. Goubran, "Perceptual Kalman filtering for speech enhancement in colored noise", 2004

20. Marcel Gabrea, "Adaptive Kalman filtering-based speech enhancement algorithm", Ecole de TechnologieSuperieure, Vol. 3, Issue 7, 2004

21. Sandeep and K. Samprasad, "A Robust Speech Enhancement by using Adaptive Kalman Filtering Algorithm", International Journal of Engineering Research and Applications (IJERA) Vol. 2, Issue 4, August 2012.

22. N. Nithiyanandam, K. Venkatesh, M. Rajesh, Transfer The Levels Of The Monitored Carbon, Nitrogen Gases From The Industries, International Journal of Recent Technology and Engineering, Volume-7 Issue-6S3 April, 2019.

23. Sivanesh Kumar, A., Brittoraj, S., Rajesh, M., Implementation of RFID with internet of things, Journal of Recent Technology and Engineering, Volume-7 Issue-6S3 April, 2019.

24. Rajesh, M., Sairam, R., Big data and health care system using mlearningJournal of Recent Technology and Engineering, Volume-7 Issue-6S3 April, 2019.

25. Rajesh, M., and J. M. Gnanasekar. "Path Observation Based Physical Routing Protocol for Wireless Ad Hoc Networks." Wireless Personal Communications 97.1 (2017): 1267-1289. 